\title{
Evidence for a Circumnuclear Torus in Edge-On Radio Galaxies
}

\author{
A. B. Peck \\ MPIfR, Auf dem Hügel 69, Bonn, Germany \\ G. B. Taylor \\ NRAO, P.O. Box O, Socorro, NM 87801, USA
}

\begin{abstract}
We have used VLBI HI absorption imaging to search for evidence for a circumnuclear atomic structure in five radio galaxies. Of these, we have found that two, (PKS 2322-123 and 1946+708), are very symmetric on parsec scales and exhibit strong evidence for a circumnuclear torus, confirming the prediction of the unified scheme. The two nearby extended radio galaxies NGC 5128 and NGC 315, on the other hand, have asymmetric core-jet morphologies on parsec scales, and their HI absorption profiles appear to arise from gas in the host galaxy which is not associated with the AGN at all. This is also consistent with the unified scheme of AGN. One remaining source, NGC 3894, also has symmetric jets, but has a very complicated Hi structure which changes on parsec scales. This source requires further study.
\end{abstract}

\section{Results}

The sources in this study strongly support the circumnuclear torus model. Although no molecular absorption has yet been detected, the signature of an extended $\mathrm{HI}$ torus has been found in PKS 2322-123 (Figure 1) and 1946+708 (Figure 2), two sources that have symmetric jets on mas scales implying nearly edge-on disks. This signature consists of a localized region of HI absorption, centered on the core of the radio source, which is orders of magnitude broader in velocity than absorption which would arise in the host galaxy. In the case of $1946+708$, this model is also supported by the detection of a region of free-free absorption.

We can compare these very broad absorption lines to the "core-jet" sources such as NGC 315 and NGC 5128 (Cen A), where it appears that the Hi absorption is taking place in colder gas in a kpc-scale disk or elsewhere in the host galaxy (Peck 1999). In these cases, we see narrow lines which are distinct from one another. The difference in the profiles between the "narrow line" and "broad line" sources suggests that the HI gas in PKS 2322-123, 1946+708, and possibly NGC 3894 , lies in a considerably more energetic environment, such as that found within a few hundred pc of an AGN. The variations in the column densities over regions on the order of $10 \mathrm{pc}$ are also consistent with this interpretation. 

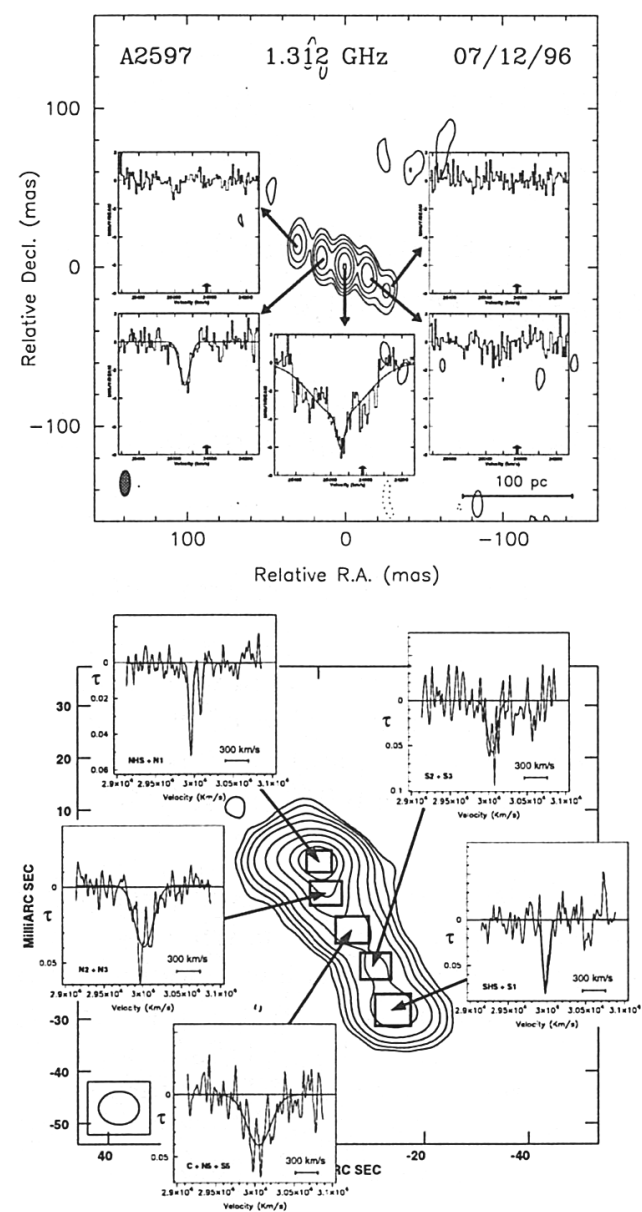

Figure 1. PKS 2232-123. Hi absorption is seen toward only two central continuum components. An extremely broad line $\left(735 \mathrm{~km} \mathrm{~s}^{-1} \mathrm{FWHM}\right)$ is seen toward the core of the source. A narrower line (110 $\left.\mathrm{km} \mathrm{s}^{-1} \mathrm{FWHM}\right)$ appears toward the core and the innermost eastern component. If the gas in this source is distributed in a disk centered on the core then it must be fairly thin $(<20 \mathrm{pc}$ ) (Taylor et al. 1999).

Figure 2. $1946+708$. The FWHM linewidth of the broad absorption component toward the core is $256 \pm 15 \mathrm{~km} \mathrm{~s}^{-1}$. The narrow lines toward the northern hotspot are probably due to small clouds of $\mathrm{HI}$ associated with an extended "clumpy" torus of warm gas. The high velocity dispersion and column density toward the core of the source, however, are indicative of fast moving circumnuclear gas, probably in a rotating circumnuclear structure (Peck, Taylor \& Conway 1999).

\section{References}

Peck, A. B., Taylor, G. B., \& Conway, J. E. 1999, ApJ, 521, 103

Peck, A. B. 1999, PhD Thesis, New Mexico Inst. of Mining and Technology.

Taylor, G. B., O'Dea, C. P., Peck, A. B. \& Koekemoer, A. M. 1999, ApJ, 512, L27 